\title{
SQUARE ROOTS IN FINITE FULL TRANSFORMATION SEMIGROUPS
}

\author{
by MARY SNOWDEN and J. M. HOWIE
}

(Received 18 December, 1980)

Let $X$ be a finite set and let $\mathscr{T}(X)$ be the full transformation semigroup on $X$, i.e. the set of all mappings from $X$ into $X$, the semigroup operation being composition of mappings. This paper aims to characterize those elements of $\mathscr{T}(X)$ which have square roots. An easily verifiable necessary condition, that of being quasi-square, is found in Theorem 2, and in Theorems 4 and 5 we find necessary and sufficient conditions for certain special elements of $\mathscr{T}(X)$. The property of being compatibly amenable is shown in Theorem 7 to be equivalent for all elements of $\mathscr{T}(X)$ to the possession of a square root.

The work reported here formed part of an M.Sc. Thesis [4] submitted to the University of St Andrews in 1979 by the first-named author.

1. Preliminaries. For unexplained terms in semigroup theory see [2]. Mapping symbols will be written on the right. We shall denote the range of a mapping $\alpha: X \rightarrow X$ by $X \alpha$ or by $\operatorname{ran} \alpha$, and by $\alpha{ }^{\circ} \alpha^{-1}$ we shall mean the equivalence relation

$$
\{(x, y) \in X \times X: x \alpha=y \alpha\} \text {. }
$$

If $|X|=n$ then the $\mathscr{J}$-classes of $\mathscr{T}(X)$ are $J^{(1)}, \ldots, J^{(n)}$, where

$$
J^{(r)}=\{\alpha \in \mathscr{T}(X):|X \alpha|=r\}
$$

is the set of mappings of rank $r$. (See [2, Ex.II.10].) Clearly

$$
J^{(1)}<J^{(2)}<\ldots<J^{(n)} .
$$

The principal factors of $\mathscr{T}(X)$ are thus $J^{(1)}$ (completely simple) and $J^{(r)} \cup\{0\}, r=2, \ldots, n$ (completely 0-simple).

Notice that $J^{(n)}=\mathscr{G}(X)$, the symmetric group on $X$. Since

$$
J_{\alpha^{2}} \leqslant J_{\alpha}
$$

for each $\alpha$ in $\mathscr{T}(X)$ it follows that an element of $J^{(n)}$ has a square root in $\mathscr{T}(X)$ if and only if it has a square root in $J^{(n)}$. Thus the characterization of squares in $J^{(n)}$ is a purely group theoretical problem. It is solved by the following theorem, which is deducible from the results of Isaacs [3], but which is proved here for completeness.

Theorem 1. Let $X$ be a finite set. An element $\alpha$ of $\mathscr{G}(X)$ is a square if and only if for each even number $k$ the decomposition of $\alpha$ into disjoint cycles involves an even number of cycles of length $k$.

Proof. Disjoint cycles, since they operate non-trivially on disjoint subsets of $X$, commute with each other. Thus if $C_{1}, \ldots, C_{r}$ are disjoint cycles then

$$
\left(C_{1} \ldots C_{r}\right)^{2}=C_{1}^{2} \ldots C_{r}^{2} \text {. }
$$

Glasgow Math. J. 23 (1982) 137-149. 
Suppose now that $\alpha$ in $\mathscr{G}(X)$ has a square root $\beta$ in $\mathscr{G}(X)$. Writing $\beta$ as $C_{1} \ldots C_{r}$, a product of disjoint cycles, we then have that

$$
\alpha=\beta^{2}=C_{1}^{2} \ldots C_{r}^{2} \text {. }
$$

If we write the cycle $C_{i}$ as $\left(a_{1} a_{2} \ldots a_{l}\right)$ then if $l$ is odd we have

$$
C_{i}^{2}=\left(a_{1} a_{3} \ldots a_{1} a_{2} a_{4} \ldots a_{1-1}\right) \text {, }
$$

a cycle of (odd) length $l$; while if $l$ is even then

$$
C_{i}^{2}=\left(a_{1} a_{3} \ldots a_{1-1}\right)\left(a_{2} a_{4} \ldots a_{1}\right),
$$

a product of two disjoint cycles of equal length $l / 2$. The decomposition of $\alpha$ into disjoint cycles is obtained by decomposing each of $C_{1}^{2}, \ldots, C_{r}^{2}$, and cycles of even length arise only when $C_{i}$ is of length divisible by 4 . The effect is that even cycles occur in pairs of equal length, so that $\alpha$ has the property described in the theorem.

Conversely, suppose that in the disjoint cycle decomposition of $\alpha$ there is an even number of cycles of each even length $k$. Each odd cycle $\left(a_{1} a_{2} \ldots a_{2 k+1}\right)$ is a square, since

$$
\left(a_{1} a_{2} \ldots a_{2 k+1}\right)=\left(a_{1} a_{k+2} a_{2} a_{k+3} \ldots a_{k} a_{2 k+1} a_{k+1}\right)^{2} .
$$

Also, the product of two disjoint cycles of equal even length $l$ is a square:

$$
\left(a_{1} a_{2} \ldots a_{l}\right)\left(b_{1} b_{2} \ldots b_{l}\right)=\left(a_{1} b_{1} a_{2} b_{2} \ldots a_{l} b_{l}\right)^{2} .
$$

By these devices one expresses $\alpha$ as a product $C_{1}^{2} \ldots C_{t}^{2}$, where $C_{1}, \ldots, C_{t}$ are disjoint cycles. Thus $\alpha=\beta^{2}$, where $\beta=C_{1} \ldots C_{r}$. This completes the proof.

In analysing elements of $\mathscr{T}(X)$ it is useful to observe that since $X$ is finite the descent

$$
X \supseteq X \alpha \supseteq X \alpha^{2} \supseteq \ldots
$$

cannot continue indefinitely. Evidently if $X \alpha^{i}=X \alpha^{i+1}$ then $X \alpha^{i+1}=X \alpha^{i+2}$; hence there exists $k \geqslant 0$ such that

$$
X \supset X \alpha \supset \ldots \supset X \alpha^{k}=X \alpha^{k+1}=X \alpha^{k+2}=\ldots
$$

Let us refer to $k$ as the contraction factor of $\alpha$ and write $k=\operatorname{cont} \alpha$. The subset $X \alpha^{k}$ will be called the stable range of $\alpha$ and will be written as $\operatorname{stran} \alpha$. If cont $\alpha=0$ then $X=X \alpha$ and so $\alpha$ is a permutation of $X$. Elements $\alpha$ such that cont $\alpha=1$ occur in all $J$-classes $J^{(r)}$ with $r<n(=|X|)$ : for such elements, $\alpha \mid X \alpha$ is a permutation of $X \alpha$. In all cases $\alpha \mid \operatorname{stran} \alpha$ is a permutation of $\operatorname{stran} \alpha$. We say that $\alpha$ is a quasi-square element of $\mathscr{T}(X)$ if the permutation $\alpha \mid \operatorname{stran} \alpha$ has a square root in the symmetric group $\mathscr{G}(\operatorname{stran} \alpha)$.

2. Quasi-square elements. We begin by proving

Lemma 1. Let $\alpha \in J^{(r)} \subseteq \mathscr{T}(X) \backslash \mathscr{G}(X)$ and suppose that cont $\alpha=1$. Let $k \geqslant 2$ be an integer. If there exists $\beta$ in $\mathscr{T}(X)$ such that $\beta^{k}=\alpha$ then $\beta \mid \operatorname{ran} \alpha$ is a permutation of $\operatorname{ran} \alpha$ and $(\beta \mid \operatorname{ran} \alpha)^{k}=\alpha \mid \operatorname{ran} \alpha$. 
Proof. Let $\operatorname{ran} \alpha=\left\{a_{1}, \ldots, a_{r}\right\}$. Since cont $\alpha=1$ we have that $\alpha \mid \operatorname{ran} \alpha$ is a permutation. Thus

$$
a_{i} \alpha=a_{i \mu} \quad(i=1, \ldots, r),
$$

where $\mu$ is a permutation of $\{1, \ldots, r\}$. If we write $a_{i \mu} \alpha^{-1}=A_{i}(i=1, \ldots, r)$, we have $a_{i} \in A_{i}$ and we may write

$$
\alpha=\left(\begin{array}{c}
A_{1}, \ldots, A_{r} \\
a_{1 \mu}, \ldots, a_{r \mu}
\end{array}\right) .
$$

If $\beta^{k}=\alpha$ then $\beta$ must be of rank $s \geqslant r$ and $\beta \circ \beta^{-1} \subseteq \alpha \circ \alpha^{-1}$. Since $a_{i}\left(\alpha \circ \alpha^{-1}\right)=A_{i}$ we have

$$
a_{i}\left(\beta \circ \beta^{-1}\right)=B_{i} \subseteq A_{i} \quad(i=1, \ldots, r) .
$$

If $a_{i} \beta\left(=B_{i} \beta\right)=b_{i}(i=1, \ldots, r)$, then

$$
b_{i} \beta^{k-1}=a_{i} \beta^{k}=a_{i} \alpha=a_{i \mu} .
$$

Hence, for $i=1, \ldots, r$,

$$
a_{i}=b_{i \mu-1} \beta^{k-1},
$$

and so

$$
b_{i}=a_{i} \beta=b_{i \mu^{-1}} \beta^{k}=b_{i \mu^{-1}} \alpha \in\left\{a_{1}, \ldots, a_{r}\right\} .
$$

Thus $\left\{b_{1}, \ldots, b_{r}\right\} \subseteq\left\{a_{1}, \ldots, a_{r}\right\}$, and since $b_{1}, \ldots, b_{r}$ are all distinct the two sets are in fact equal. Hence $\beta \mid \operatorname{ran} \alpha$ is a permutation of $\operatorname{ran} \alpha=\left\{a_{1}, \ldots, a_{r}\right\}$. Since $\beta^{k}=\alpha$ it is now obvious that

$$
(\beta \mid \operatorname{ran} \alpha)^{k}=\alpha \mid \operatorname{ran} \alpha .
$$

Theorem 2. Let $\alpha \in \mathscr{T}(X)$ where $X$ is a finite set. If $\alpha$ has a square root in $\mathscr{T}(X)$ then $\alpha$ is quasi-square.

Proof. If cont $\alpha=0$ this is trivial, while if cont $\alpha=1$ Lemma 1 shows that the existence of a square root for $\alpha$ implies that $\beta \mid \operatorname{ran} \alpha$ is a square root within $\mathscr{G}(\operatorname{ran} \alpha)$ for the permutation $\alpha \mid \operatorname{ran} \alpha$. Suppose now that cont $\alpha=k>1$ and that $\alpha$ has a square root $\beta$ in $\mathscr{T}(X)$. Then $X \alpha^{k}=X \alpha^{2 k}$ and so cont $\left(\alpha^{k}\right)=1$. Moreover $\beta^{2 k}=\alpha^{k}$ and so by Lemma 1 , $\beta \mid \operatorname{ran} \alpha^{k}$ is a permutation of $\operatorname{ran} \alpha^{k}=\operatorname{stran} \alpha$. Since $\beta^{2}=\alpha$ it is now easily seen that

$$
(\beta \mid \operatorname{stran} \alpha)^{2}=\alpha \mid \operatorname{stran} \alpha \text {. }
$$

Thus $\alpha$ is quasi-square.

The main usefulness of this theorem lies in its contrapositive form: if $\alpha$ is not quasi-square then it cannot have a square root in $\mathscr{T}(X)$. Thus, for example, if $X=$ $\{1, \ldots, 9\}$ and

$$
\alpha=\left(\begin{array}{lllllllll}
1 & 2 & 3 & 4 & 5 & 6 & 7 & 8 & 9 \\
2 & 3 & 4 & 3 & 8 & 9 & 8 & 9 & 7
\end{array}\right)
$$


then $\operatorname{stran} \alpha=X \alpha^{2}=\{3,4,7,8,9\}$ and $\alpha \mid \operatorname{stran} \alpha=(34)(789)$. By Theorem 1 this has no square root in $\mathscr{G}(\{3,4,7,8,9\})$ and so $\alpha$ has no square root in $\mathscr{T}(\{1, \ldots, 9\})$.

In the case where cont $\alpha=1$ we have a converse to Theorem 2 .

Theorem 3. Let $\alpha \in \mathscr{T}(X)$ and suppose that cont $\alpha=1$. Then $\alpha$ has a square root in $\mathscr{T}(X)$ if and only if it is quasi-square.

Proof. Half of this is included in Theorem 2. Suppose now that $\alpha$, with contraction factor 1 , is quasi-square. We may, as in the proof of Lemma 1 , write $\alpha$ as

$$
\left(\begin{array}{c}
A_{1}, \ldots, A_{r} \\
a_{1 \mu}, \ldots, a_{r \mu}
\end{array}\right)
$$

where $a_{i} \in A_{i}(i=1, \ldots, r)$ and where $\mu$ is a permutation of $\{1, \ldots, r\}$. The permutation $\alpha \mid \operatorname{ran} \alpha$ is described by $a_{i} \rightarrow a_{i \mu}(i=1, \ldots, r)$. Since $\alpha$ is quasi-square there exists a permutation $\nu$ of $\{1, \ldots, r\}$ such that $\nu^{2}=\mu$. Then the element

$$
\beta=\left(\begin{array}{l}
A_{1}, \ldots, A_{r} \\
a_{1 v}, \ldots, a_{r v}
\end{array}\right)
$$

of $\mathscr{T}(X)$ is a square root for $\alpha$.

This completes the proof of Theorem 3. Notice, however, that by [2, Ex.II.10] the square root $\beta$ obtained for $\alpha$ has the property that $\beta \mathscr{H} \alpha$; for $\alpha$ and $\beta$ have the same range $\left\{a_{1}, \ldots, a_{r}\right\}$, and $\alpha \circ \alpha^{-1}, \beta \circ \beta^{-1}$ both have equivalence classes $A_{1}, \ldots, A_{r}$. We thus have:

Corollary. Let $\alpha \in \mathscr{T}(X)$, with cont $\alpha=1$. If $\alpha$ has a square root in $\mathscr{T}(X)$ then it has a square root in its own $\mathscr{H}$-class.

3. Elements in extremal $\mathscr{J}$-classes. We have seen (Theorem 1) how to recognise squares in the top $\mathscr{g}$-class $J^{(n)}$. The bottom $\mathscr{J}$-class $J^{(1)}$ presents no problem at all. It consists of all the constant mappings of $X$ and is a right zero semigroup. Every element is its own square root.

We now examine the $\mathscr{g}$-classes $J^{(n-1)}$ and $J^{(2)}$.

THEOREM 4. Let $|X|=n \geqslant 3$ and let $\alpha \in J^{(n-1)}$. Then $\alpha$ has a square root in $\mathscr{T}(X)$ if and only if $\alpha$ is quasi-square and cont $\alpha=1$.

Proof. In view of Theorem 3 we require to show only that if $\alpha \in J^{(n-1)}$ and cont $\alpha>1$ then $\alpha$ has no square root. A square root $\beta$ for such an $\alpha$ would have to lie in $J^{(n-1)}$, since $\beta \in J^{(n)}$ implies $\beta^{\overline{2}} \in J^{(n)}$. If cont $\beta=1$ then $X \beta^{2}=X \beta^{4}$; hence $\operatorname{cont}\left(\beta^{2}\right)=1$ and so $\beta^{2}$ cannot equal $\alpha$. On the other hand if cont $\beta>1$ then $X \beta^{2} \subset X \beta$ and so

$$
J_{\beta^{2}}<J_{\beta}=J^{(n-1)}
$$

hence again $\beta^{2}$ cannot equal $\alpha$, and so no square root of $\alpha$ can exist. 
Theorem 5. Let $|X|=n \geqslant 4$ and let $\alpha \in J^{(2)}$. Then $\alpha$ has a square root in $\mathscr{T}(X)$ if and only if either (i) $\alpha$ is idempotent or (ii) cont $\alpha=2$ and $\operatorname{ran} \alpha$ is contained in an $\left(\alpha^{\circ} \alpha^{-1}\right)$ class containing at least 3 elements.

Proof. If cont $\alpha=1$ we may write

$$
\alpha=\left(\begin{array}{ll}
A_{1} & A_{2} \\
a_{1 \mu} & a_{2 \mu}
\end{array}\right),
$$

where $\mu$ is a permutation of $\{1,2\}$ and where $a_{1} \in A_{1}, a_{2} \in A_{2}$. If $\alpha$ is to be quasi-square then $\mu$ must be the identical permutation and so

$$
\alpha=\left(\begin{array}{cc}
A_{1} & A_{2} \\
a_{1} & a_{2}
\end{array}\right)
$$

an idempotent. It is now obvious that if $\alpha \in J^{(2)}$ and cont $\alpha=1$ then $\alpha$ has a square root if and only if it is idempotent.

Now suppose that cont $\alpha=2$; this is the only other possibility since $X \alpha \supset X \alpha^{2}$ implies $\left|X \alpha^{2}\right|=1$ and no further descent is possible. Writing

$$
\alpha=\left(\begin{array}{cc}
A_{1} & A_{2} \\
x_{1} & x_{2}
\end{array}\right)
$$

with $x_{1}, x_{2} \in A_{1}$, we suppose that $\left|A_{1}\right| \geqslant 3$. Choose $y$ in $A_{1} \backslash\left\{x_{1}, x_{2}\right\}$ and let

then $\beta^{2}=\alpha$.

$$
\beta=\left(\begin{array}{ccc}
A_{1} \backslash\{y\}, & y & A_{2} \\
x_{1}, & x_{2}, & y
\end{array}\right)
$$

Conversely, suppose that cont $\alpha=2$ and that

$$
\alpha=\left(\begin{array}{cc}
A_{1} & A_{2} \\
x_{1} & x_{2}
\end{array}\right)\left(\text { with } x_{1}, x_{2} \text { in } A_{1}\right)
$$

has a square root $\beta$. Let $z \in A_{2}$ and let $z \beta=y$. Then

$$
y \beta=z \alpha=x_{2} .
$$

If $y \in A_{2}$ then $y \alpha=x_{2}$ and so

But then

$$
x_{2}=y \alpha=y \beta^{2}=x_{2} \beta
$$

$$
x_{1}=x_{2} \alpha=x_{2} \beta^{2}=x_{2} \beta=x_{2},
$$

which is a contradiction. Hence $y \in A_{1}$.

If $y=x_{1}$ then $x_{1} \beta=x_{2}$; hence

$$
x_{2} \beta=x_{1} \beta^{2}=x_{1} \alpha=x_{1}
$$

and so

$$
x_{2}=x_{1} \beta=x_{2} \beta^{2}=x_{2} \alpha=x_{1},
$$


again a contradiction. Hence $y \neq x_{1}$. If $y=x_{2}$ we have $x_{2} \beta=x_{2}$, which as we saw in the last paragraph leads to a contradiction. Hence $y \neq x_{2}$ and so there must exist $y$ in $A_{1}$ distinct from both $x_{1}$ and $x_{2}$. Thus $\left|A_{1}\right| \geqslant 3$ as required.

4. Duals; amenable elements. The results of Section 3 make it clear that Theorem 3 will not extend to elements $\alpha$ for which cont $\alpha \geqslant 2$. For example, if $X=\{1,2,3\}$ and

$$
\alpha=\left(\begin{array}{lll}
1 & 2 & 3 \\
1 & 1 & 2
\end{array}\right),
$$

then $\alpha$ is certainly quasi-square but by Theorem 4 cannot have a square root. Some further concepts are now required.

If $\alpha \in \mathscr{T}(X)$ then for each $x$ in $X$ we have a sequence

$$
x, x \alpha, x \alpha^{2}, \ldots
$$

of elements of $X$, and since $X$ is finite these elements cannot all be distinct. If $r$ is the least element of the non-empty set

$$
\left\{w \in \mathbb{N} \cup\{0\}:(\exists z>w) x \alpha^{w}=x \alpha^{z}\right\}
$$

we call $r$ the index of $x$ relative to $\alpha$ and write either $r=i_{\alpha}(x)$ or (where no confusion will arise) $r=i(x)$. Notice that $i(x) \geqslant 1$ unless $x \in \operatorname{stran} \alpha$ and that in all cases $x \alpha^{i(x)} \in \operatorname{stran} \alpha$. If $q$ is the least element of the non-empty set

$$
\left\{z \in \mathbb{N}: x \alpha^{i(x)}=x \alpha^{i(x)+z}\right\},
$$

we call $q$ the period of $x$ relative to $\alpha$ and write either $q=p_{\alpha}(x)$ or $q=p(x)$. It is always the case that $p(x) \geqslant 1$.

In attempting to find necessary and sufficient conditions for an element $\alpha$ of $\mathscr{T}(X)$ to have a square root we are led to consider what we shall call a ' $\gamma$-dual' of an element $x$ in $X \backslash \operatorname{ran} \alpha$. Because of Theorem 2 we may confine attention to quasi-square elements; i.e. we may suppose that $\alpha \mid \operatorname{stran} \alpha$ is a permutation of stran $\alpha$ having square root $\gamma$. Now define $y$ to be a $\gamma$-dual of $x$ if $y \in X \backslash \operatorname{ran} \alpha$ and one of the following four conditions is satisfied:

(D1) $i(y)=i(x)$ and $\left(x \alpha^{i(x)}\right) \gamma=y \alpha^{i(y)}$;

(D2) $i(y)=i(x)$ and $x \alpha^{i(x)}=\left(y \alpha^{i(y)}\right) \gamma$;

(D3) $i(y)=i(x)+1$ and $\left(x \alpha^{i(x)}\right) \gamma=y \alpha^{i(y)}$;

(D4) $i(y)=i(x)-1$ and $x \alpha^{i(x)}=\left(y \alpha^{i(y)}\right) \gamma$.

If it is necessary to specify more exactly the relationship of $y$ to $x$ we shall say (for example) that ' $y$ is a $\gamma$-dual of $x$ under rule (D1)'. It is easy to see that if $y$ is a $\gamma$-dual of $x$ then $x$ is a $\gamma$-dual of $y$.

We define an $\left(\alpha \circ \alpha^{-1}\right)$-class $A$ in $X$ to be basic if $A \cap \operatorname{ran} \alpha=\varnothing$, and we say that $x \in X$ is a basic element if it belongs to a basic $\left(\alpha \circ \alpha^{-1}\right)$-class, i.e. if $x\left(\alpha^{\circ} \alpha^{-1}\right) \cap \operatorname{ran} \alpha=\varnothing$. 
We say that $\alpha$ is amenable if it is quasi-square and if there exists a square root $\gamma$ of the permutation $\alpha \mid \operatorname{stran} \alpha$ such that every basic element of $X$ has a $\gamma$-dual. Then we have

TheOrem 6. Let $\alpha \in \mathscr{T}(X)$. If $\alpha$ has a square root in $\mathscr{T}(X)$ then $\alpha$ is amenable.

Proof. Let $\alpha \in \mathscr{T}(X)$ and suppose that $\alpha$ has a square root $\beta$. Let $x$ be a basic element of $X$. Then $i(x) \geqslant 2$, since $i(x) \leqslant 1$ would imply that there exists $m \geqslant 1$ such that $x \alpha=x \alpha^{m+1}$. This would give $x \alpha^{m} \in x\left(\alpha \circ \alpha^{-1}\right)$, in contradiction to the definition of a basic element.

By the definition of index and period we have

$$
x \alpha^{i(x)}=x \alpha^{i(x)+p(x)}
$$

and

$$
(\forall m \in \mathbb{N}) x \alpha^{i(x)-1} \neq x \alpha^{i(x)-1+m} .
$$

Hence

$$
x \beta^{2 i(x)}=x \beta^{2 i(x)+2 p(x)}
$$

and

$$
(\forall m \in \mathbb{N}) \quad x \beta^{2 i(x)-2} \neq x \beta^{2 i(x)-2+2 m} .
$$

Rewriting these slightly, we get

$$
(x \beta) \beta^{2 i(x)-1}=(x \beta) \beta^{2 i(x)-1+2 p(x)}
$$

and

$$
(\forall m \in \mathbb{N})(x \beta) \beta^{2 i(x)-3} \neq(x \beta) \beta^{2 i(x)-3+2 m} .
$$

It follows that

$$
(x \beta) \beta^{2 i(x)}=(x \beta) \beta^{2 i(x)+2 p(x)}
$$

and

$$
(\forall m \in \mathbb{N})(x \beta) \beta^{2 i(x)-4} \neq(x \beta) \beta^{2 i(x)-4+2 m},
$$

i.e. that

$$
(x \beta) \alpha^{i(x)}=(x \beta) \alpha^{i(x)+p(x)}
$$

and

$$
(\forall m \in \mathbb{N})(x \beta) \alpha^{i(x)-2} \neq(x \beta) \alpha^{i(x)-2+2 m} .
$$

From (A) it follows that $i_{\alpha}(x \beta) \leqslant i(x)$ and from.(B) it follows that $i_{\alpha}(x \beta)>i(x)-2$. Thus either $i(x \beta)=i(x)$ or $i(x \beta)=i(x)-1$.

We now find it convenient to consider four cases as follows:

(1) $x \beta \notin \operatorname{ran} \alpha, i(x \beta)=i(x)-1$;

(2) $x \beta \notin \operatorname{ran} \alpha, i(x \beta)=i(x)$;

(3) $x \beta \in \operatorname{ran} \alpha, i(x \beta)=i(x)-1$;

(4) $x \beta \in \operatorname{ran} \alpha, i(x \beta)=i(x)$. 
Let $\gamma=\beta \mid \operatorname{stran} \alpha$; by Lemma $1, \gamma$ is a permutation of stran $\alpha$ and $\gamma^{2}=\alpha \mid \operatorname{stran} \alpha$. Since in case (1) we have

$$
i(x \beta)=i(x)-1 \text { and } x \alpha^{i(x)}=\left[(x \beta) \alpha^{i(x)-1}\right] \gamma,
$$

the element $x \beta$ is a $\gamma$-dual for $x$ under rule (D4). Similarly in case (2) the element $x \beta$ is a $\gamma$-dual for $x$ under rule (D1).

The element $x \beta$ cannot be a $\gamma$-dual in cases (3) and (4), since one of the requirements for a $\gamma$-dual is that it belong to $X \backslash \operatorname{ran} \alpha$. In both these cases, however, there exists an element $x_{1}$ in $X$ such that $x_{1} \alpha=x \beta$. Then $x_{1} \notin \operatorname{ran} \alpha$, since if we had $x_{1}=t \alpha(t \in X)$ it would follow that

$$
x \alpha=x \beta^{2}=x_{1} \alpha \beta=t \alpha^{2} \beta=(t \beta \alpha) \alpha ;
$$

hence

$$
t \beta \alpha \in x\left(\alpha \circ \alpha^{-1}\right) \cap \operatorname{ran} \alpha,
$$

in contradiction to the hypothesis that $x$ is a basic element.

Notice next that

$$
\begin{aligned}
x_{1} \alpha^{i(x \beta)+1} & =(x \beta) \alpha^{i(x \beta)}=(x \beta) \alpha^{i(x \beta)+p(x \beta)} . \\
& =x_{1} \alpha^{i(x \beta)+1+p(x \beta)},
\end{aligned}
$$

so that $i\left(x_{1}\right) \leqslant i(x \beta)+1$. This is in fact an equality, since if

$$
x_{1} \alpha^{r}=x_{1} \alpha^{r+m} \quad(m>0)
$$

then $r \geqslant 1$ and so

$$
(x \beta) \alpha^{r-1}=(x \beta) \alpha^{r-1+m} .
$$

By the definition of $i(x \beta)$ it then follows that $r-1 \geqslant i(x \beta)$. Hence $r \geqslant i(x \beta)+1$ and it is now clear that

$$
i\left(x_{1}\right)=i(x \beta)+1
$$

In case (3) we now have

$$
i\left(x_{1}\right)=i(x) \text { and } x \alpha^{i(x)}=\left(x_{1} \alpha^{i(x)}\right) \gamma
$$

so that $x_{1}$ is a $\gamma$-dual of $x$ under rule (D2). Similarly in case (4) we find that $x_{1}$ is a $\gamma$-dual of $x$ under rule (D3). Thus $\alpha$ is amenable.

5. Compatibly amenable elements. The refined necessary condition for the existence of a square root given by Theorem 6 is unfortunately still not sufficient. We shall eventually give an example, but this will be easier to explain if we defer it until we have introduced some further concepts. Let us begin by noticing that in checking for amenability we do not need to check every basic element, for if $x$ and $x^{\prime}$ are basic elements in the same basic $\left(\alpha^{\circ} \alpha^{-1}\right)$-class then $x \alpha=x^{\prime} \alpha$; hence $i(x)=i\left(x^{\prime}\right)(\geqslant 2)$ and any element $y$ of $X \backslash \operatorname{ran} \alpha$ that is a $\gamma$-dual for $x$ is also a $\gamma$-dual for $x^{\prime}$. It is thus sufficient to check that $\gamma$-duals exist for the elements in a cross-section of the basic $\left(\alpha^{\circ} \alpha^{-1}\right)$-classes. 
Suppose now that $\alpha$ is an amenable element of $\mathscr{T}(X)$, and choose a cross-section Bas $\alpha$ of the basic $\left(\alpha^{\circ} \alpha^{-1}\right)$-classes. We can express the amenability of $\alpha$ by saying that for some square root $\gamma$ of the permutation $\alpha \mid \operatorname{stran} \alpha$ there is a mapping $\Delta: \operatorname{Bas} \alpha \rightarrow X \backslash \operatorname{ran} \alpha$ associating each $x$ in Bas $\alpha$ with a $\gamma$-dual $x \Delta$ in $X \backslash \operatorname{ran} \alpha$. We shall refer to $\Delta$ as a dualizar mapping. If $(y, z) \in \Delta$ (i.e. if $y \in \operatorname{Bas} \alpha$ and $z=y \Delta$ ) define

$$
\begin{aligned}
& (y, z)^{(1)}=\left\{(y, z),(z, y \alpha),(y \alpha, z \alpha),\left(z \alpha, y \alpha^{2}\right), \ldots\right\}, \\
& (y, z)^{(2)}=\left\{(z, y),(y, z \alpha),(z \alpha, y \alpha),\left(y \alpha, z \alpha^{2}\right), \ldots\right\} .
\end{aligned}
$$

Both these sets are in fact finite, with size dependent on the index and period of $y$ and $z$. For each subset $A$ of $\Delta$ we define

$$
\Delta_{\mathrm{A}}=\bigcup_{(y, z) \in \mathrm{A}}(y, z)^{(1)} \cup \underset{(y, z) \in \Delta \backslash \mathrm{A}}{\bigcup}(y, z)^{(2)},
$$

and we say that $\Delta$ is a compatible dualizer mapping if for some $A \subseteq \Delta$ the relation $\Delta_{A}$ is a partial mapping, i.e. if $\Delta_{\mathrm{A}}$ satisfies

$$
(v, w) \in \Delta_{\mathrm{A}} \quad \text { and } \quad\left(v, w^{\prime}\right) \in \Delta_{\mathrm{A}} \Rightarrow w=w^{\prime} .
$$

We say that an element $\alpha$ of $\mathscr{T}(X)$ is compatibly amenable if it is amenable and if there exists a compatible dualizer mapping.

Before describing an example of an amenable element that is not compatibly amenable, we establish two fairly easy properties of compatible dualizer mappings.

Lemma 2. If $\Delta: \operatorname{Bas} \alpha \rightarrow X \backslash \operatorname{ran} \alpha$ is a compatible dualizer mapping, then $\Delta$ is one-one.

Proof. Suppose that $(x, z),(y, z) \in \Delta$, where $x, y \in \operatorname{Bas} \alpha$. Since $\Delta$ is compatible, there exists $A \subseteq \Delta$ for which $\Delta_{A}$ is a partial mapping. On the face of it there are four possibilities:

(i) $(x, z) \in A,(y, z) \in A$;

(ii) $(x, z) \in A,(y, z) \in \Delta \backslash A$;

(iii) $(x, z) \in \Delta \backslash A,(y, z) \in A$;

(iv) $(x, z) \in \Delta \backslash A,(y, z) \in \Delta \backslash A$.

In case (i) we have, by definition of $\Delta_{\mathrm{A}}$,

$$
(z, x \alpha),(z, y \alpha) \in \Delta_{\mathrm{A}} .
$$

Hence, by the partial mapping property, $x \alpha=y \alpha$; hence, since $x, y$ are members of a cross-section of $\left(\alpha \circ \alpha^{-1}\right)$-classes, $x=y$.

Case (ii) cannot arise, since we have $(x, z),(z, y) \in \Delta_{\mathrm{A}}$ and hence $y=x \alpha \in \operatorname{ran} \alpha$, a contradiction. A similar argument shows that case (iii) is impossible. In case (iv) we have $(z, x),(z, y) \in \Delta_{\mathrm{A}}$ and so $x=y$.

Lemma 3. Suppose that $x_{1}, x_{2}, y_{1}, y_{2}$ are distinct elements of Bas $\alpha$ such that each of $y_{1}, y_{2}$ is a $\gamma$-dual for each of $x_{1}, x_{2}$. If the $\gamma$-dualizer mapping $\Delta$ contains the pairs $\left(x_{1}, y_{1}\right)$, $\left(x_{2}, y_{2}\right),\left(y_{1}, x_{2}\right),\left(y_{2}, x_{1}\right)$, then it is not compatible. 
Proof. Suppose by way of contradiction that $\Delta$ is compatible, i.e. that there exists $A \subseteq \Delta$ for which $\Delta_{A}$ is a partial mapping. Suppose first that $\left(x_{1}, y_{1}\right) \in A$. Then $\left(x_{1}, y_{1}\right) \in \Delta_{A}$ and so $\left(y_{2}, x_{1}\right) \in A$ (since otherwise $\left(x_{1}, y_{2}\right) \in \Delta_{A}$ in contradiction to the partial mapping property). Hence $\left(x_{1}, y_{2} \alpha\right) \in \Delta_{\mathrm{A}}$ and so $y_{1}=y_{2} \alpha$. This is a contradiction since $y_{1} \in \mathrm{Bas} \alpha \subseteq$ $X \backslash \operatorname{ran} \alpha$.

Hence $\left(x_{1}, y_{1}\right) \notin A$ and so $\left(y_{1}, x_{1}\right) \in \Delta_{A}$. But this implies $\left(y_{1}, x_{2}\right) \notin A$ (since otherwise $\left(y_{1}, x_{2}\right) \in \Delta_{A}$ and the partial mapping condition is violated). Hence $\left(y_{1}, x_{2} \alpha\right) \in \Delta_{A}$ and so $x_{1}=x_{2} \alpha$, again a contradiction, since $x_{1} \in \operatorname{Bas} \alpha$. This completes the proof.

Example. Let $X=\{1, \ldots, 13\}$ and let

$$
\alpha=\left(\begin{array}{ccccccccc}
\{1,2\}, & \{3,4\}, & \{5,6\}, & \{7,8\}, & 9, & 10, & 11, & 12, & 13 \\
5, & 1, & 3, & 2, & 7, & 8, & 4, & 6, & 11
\end{array}\right)
$$

Here $\operatorname{stran} \alpha=X \alpha^{3}=\{1,3,5\}$ and $\alpha \mid \operatorname{stran} \alpha=(153)$, a cycle of length 3 . This has the unique square root $\gamma=(135)$ and so $\alpha$ is quasi-square. The basic $\left(\alpha \circ \alpha^{-1}\right)$-classes are

$$
\{9\},\{10\},\{12\},\{13\}
$$

and so the only possible choice for $\operatorname{Bas} \alpha$ is $\{9,10,12,13\}$. Notice that in this case we have Bas $\alpha=X \backslash \operatorname{ran} \alpha$.

About the basic elements we summarise the relevant information in a table:

$\begin{array}{rccc}x & i(x) & x \alpha^{i(x)} & \left(x \alpha^{i(x)}\right) \gamma \\ 9 & 3 & 5 & 1 \\ 10 & 3 & 5 & 1 \\ 12 & 2 & 3 & 5 \\ 13 & 3 & 1 & 3\end{array}$

Possible $\gamma$-duals of 9 are 12 (rule (D4)) and 13 (rule (D1)); possible $\gamma$-duals of 10 are 12 (rule (D4)) and 13 (rule (D2)). Hence, bearing in mind the restrictions imposed by Lemmas 2 and 3, we see that the only possible compatible dualizer mappings are

$$
\begin{aligned}
& \Delta^{(1)}=\{(9,12),(10,13),(13,10),(12,9)\}, \\
& \Delta^{(2)}=\{(9,13),(10,12),(12,10),(13,9)\} .
\end{aligned}
$$

For brevity let us now write

$$
K=\{(2,1),(1,5),(5,5),(5,3),(3,3),(3,1),(1,1)\} .
$$

Routine calculation show that

$$
\begin{aligned}
(9,12)^{(1)} & =\{(9,12),(12,7),(7,6),(6,2),(2,3),(3,5),(5,1),(1,3)\} \\
(9,12)^{(2)} & =\{(12,9),(9,6),(6,7),(7,3),(3,2)\} \cup K \\
(9,13)^{(1)} & =\{(9,13),(13,7),(7,11),(11,2),(2,4),(4,5),(5,1),(1,3),(3,5)\} \\
(9,13)^{(2)} & =\{(13,9),(9,11),(11,7),(7,4),(4,2)\} \cup K \\
(10,12)^{(1)} & =\{(10,12),(12,8),(8,6),(6,2),(2,3),(3,5),(5,1),(1,3)\} \\
(10,12)^{(2)} & =\{(12,10),(10,6),(6,8),(8,3),(3,2)\} \cup K \\
(10,13)^{(1)} & =\{(10,13),(13,8),(8,11),(11,2),(2,4),(4,5),(5,1),(1,3),(3,5)\} \\
(10,13)^{(2)} & =\{(13,10),(10,11),(11,8),(8,4),(4,2)\} \cup K .
\end{aligned}
$$


Hence, since $(9,12)^{(2)}\left(=(12,9)^{(1)}\right),(9,13)^{(2)},(10,12)^{(2)}$ and $(10,13)^{(2)}$ all lack the partial mapping property, the only possible choice for $A \subseteq \Delta^{(1)}$ giving a partial mapping $\Delta_{A}^{(1)}$ is

$$
A=\{(9,12),(10,13)\} \text {. }
$$

But even this does not work, since examination of $(9,12)^{(1)}$ and $(10,13)^{(1)}$ shows that $(2,3),(2,4) \in \Delta_{A}^{(1)}$. Thus $\Delta^{(1)}$ is not a compatible dualizer mapping.

Similarly, for $\Delta^{(2)}$ the only subset $A$ that has any chance of providing a partial mapping $\Delta_{A}^{(2)}$ is

$$
A=\{(9,13),(10,12)\}
$$

and this fails since $(2,3),(2,4) \in \Delta_{A}^{(2)}$. We conclude that $\alpha$ is amenable but not compatibly amenable.

Hence $\alpha$ has no square root, by virtue of our final theorem:

THEOREM 7. Let $X$ be a finite set and let $\alpha$ be an element of the full transformation semigroup $\mathscr{T}(X)$. Then $\alpha$ has a square root in $\mathscr{T}(X)$ if and only if it is compatibly amenable.

Proof. Suppose first that $\alpha$ has a square $\operatorname{root} \beta$. Then $\alpha$ is amenable, by Theorem 6 . Recalling the method of proof of that theorem, we return to the four cases there considered. Effectively we divided Bas $\alpha$ into four disjoint subsets $A_{1}, \ldots, A_{4}$ :

$$
\begin{aligned}
& A_{1}=\{x \in \operatorname{Bas} \alpha: x \beta \notin \operatorname{ran} \alpha, i(x \beta)=i(x)-1\}, \\
& A_{2}=\{x \in \operatorname{Bas} \alpha: x \beta \notin \operatorname{ran} \alpha, i(x \beta)=i(x)\}, \\
& A_{3}=\{x \in \operatorname{Bas} \alpha: x \beta \in \operatorname{ran} \alpha, i(x \beta)=i(x)-1\}, \\
& A_{4}=\{x \in \operatorname{Bas} \alpha: x \beta \in \operatorname{ran} \alpha, i(x \beta)=i(x)\} .
\end{aligned}
$$

We defined $\gamma$ as $\beta \mid$ stran $\alpha$ and chose $x \beta$ as $\gamma$-dual of $x$ if $x \in A_{1} \cup A_{2}$. If $x \in A_{3} \cup A_{4}$ we introduced a new element $x_{1}$ such that $x_{1} \alpha=x \beta$ and took $x_{1}$ as $\gamma$-dual of $x$. We do not change this latter procedure, but in order to ensure that our dualizer mapping $\Delta$ is compatible we need to be a little more careful in choosing the $\gamma$-dual of an element of $A_{1} \cup A_{2}$. It may be that $x \beta \notin \operatorname{Bas} \alpha$ but that there exists an element $z$ in $(x \beta)\left(\beta \circ \beta^{-1}\right) \cap$ Bas $\alpha$. There certainly will not exist more than one such $z$, since two distinct elements of Bas $\alpha$ cannot be $\left(\alpha \circ \alpha^{-1}\right)$-equivalent. But if there does exist such a $z$ we choose it rather than $x \beta$ to be the $\gamma$-dual of $x$. Since $(x \beta) \beta^{m}=z \beta^{m}$ for all $m \geqslant 1$ it certainly serves as well. If

$$
(x \beta)\left(\beta \circ \beta^{-1}\right) \cap \operatorname{Bas} \alpha=\varnothing
$$

we choose $\gamma$-dual $x \beta$ as before. Notice that for all $x$ in $A_{1} \cup A_{2}$ and for all $m \geqslant 1$ we have

$$
(x \Delta) \alpha^{m}=x \beta \alpha^{m}=x \alpha^{m} \beta ;
$$

also,

$$
(x \Delta) \beta=x \alpha .
$$

Now let $A=\left\{(x, x \Delta): x \in A_{1} \cup A_{2}\right\}$, so that

$$
\Delta_{A}=\bigcup_{x \in A_{1} \cup A_{2}}(x, x \Delta)^{(1)} \cup \bigcup_{x \in A_{3} \cup A_{4}}\left(x, x_{1}\right)^{(2)} .
$$


We show that $\Delta_{\mathrm{A}}$ is a partial mapping.

For each $y$ in $A_{1} \cup A_{2}$,

$$
(y, y \Delta)^{(1)}=\left\{(y, y \Delta),(y \Delta, y \alpha),(y \alpha, y \alpha \beta),\left(y \alpha \beta, y \alpha^{2}\right), \ldots,\left(y \alpha^{l}, y \alpha^{\prime} \beta\right), \ldots\right\},
$$

and for each $z$ in $A_{3} \cup A_{4}$,

$$
\left(z, z_{1}\right)^{(2)}=\left\{\left(z_{1}, z\right),(z, z \beta),(z \beta, z \alpha),(z \alpha, z \alpha \beta), \ldots,\left(z \alpha^{l}, z \alpha^{\prime} \beta\right), \ldots\right\} .
$$

Thus for the most part the relation $\Delta_{A}$ coincides with the function $\beta$. The only exceptions are some elements $y$ in $A_{1} \cup A_{2}$, and elements $z_{1}\left(z \in A_{3} \cup A_{4}\right)$. If $y \in A_{1} \cup A_{2}$ and $(y \beta)\left(\beta \circ \beta^{-1}\right) \cap B a s \alpha \neq \varnothing$ then $y \Delta \in A_{3} \cup A_{4}$. Thus

and

$$
(y \Delta, y \alpha) \in(y, y \Delta)^{(1)} \subseteq \Delta_{A}
$$

$$
(y \Delta,(y \Delta) \beta) \in\left(y \Delta,(y \Delta)_{1}\right)^{(2)} \subseteq \Delta_{\mathbf{A}} .
$$

Since $(y \Delta) \beta=y \alpha$ this does not destroy the partial mapping property of $\Delta_{\mathrm{A}}$.

If $z \in A_{3} \cup A_{4}$ we may have $z_{1} \in A_{1} \cup A_{2}$. But in that case the set $\left(z_{1} \beta\right)\left(\beta \circ \beta^{-1}\right) \cap$ Bas $\alpha$ contains the (necessarily unique) element $z$ and so the pair $\left(z_{1}, z_{1} \Delta\right)$ in $\left(z_{1}, z_{1} \Delta\right)^{(1)}$ coincides with the pair $\left(z_{1}, z\right)$ in $\left(z, z_{1}\right)^{(2)}$. We conclude that $\Delta_{\mathrm{A}}$ is a partial mapping and thus that $\alpha$ is compatibly amenable.

Now suppose that $\alpha$ is compatibly amenable, with $\gamma$ as square root of $\alpha \mid \operatorname{stran} \alpha$, with Bas $\alpha$ as the chosen cross-section of the basic $\left(\alpha \circ \alpha^{-1}\right)$-classes, with $\Delta$ as compatible dualizer mapping and with subset $A$ of $\Delta$ such that $\Delta_{A}$ is a partial mapping. We find it convenient to partition $X$ into three disjoint subsets, $P, Q, R$ as follows:

$$
\begin{aligned}
& P=\bigcup_{r=0}^{\infty}[\text { Bas } \alpha \cup(\operatorname{Bas} \alpha) \Delta] \alpha^{r}, \\
& Q=\operatorname{stran} \alpha \backslash(P \cap \operatorname{stran} \alpha), \\
& R=X \backslash(P \cup Q) .
\end{aligned}
$$

Notice that if $x \in \operatorname{ran} \alpha \backslash \operatorname{stran} \alpha$ then $x \in P$; for either $x \in(\operatorname{Bas} \alpha) \alpha$ or there exists $x_{1} \in \operatorname{ran} \alpha$ such that $x_{1} \alpha=x$; then either $x_{1} \in(\operatorname{Bas} \alpha) \alpha$, giving $x \in(\operatorname{Bas} \alpha) \alpha^{2}$, or there exists $x_{2} \in \operatorname{ran} \alpha$ such that $x_{2} \alpha=x$; and so on. Thus $\operatorname{ran} \alpha \subseteq P \cup Q$ and so $R$ consists of those elements in $X \backslash \operatorname{ran} \alpha$ not in Bas $\alpha \cup(\operatorname{Bas} \alpha) \Delta$. If $x \in R$ then either

$$
x\left(\alpha \circ \alpha^{-1}\right) \cap \operatorname{Bas} \alpha \neq \varnothing
$$

(which happens if $x\left(\alpha^{\circ} \alpha^{-1}\right)$ is a basic $\left(\alpha^{\circ} \alpha^{-1}\right)$-class) or $x\left(\alpha^{\circ} \alpha^{-1}\right)$ intersects with ran $\alpha$. Thus either $x\left(\alpha^{\circ} \alpha^{-1}\right) \cap P \neq \varnothing$ or $x\left(\alpha \circ \alpha^{-1}\right) \cap Q \neq \varnothing$.

From the definitions of $P$ and $\Delta_{A}$ it is clear that dom $\Delta_{A}=P$. We now define a mapping $\beta: X \rightarrow X$ as follows: if $x \in P$ let $x \beta=x \Delta_{A}$; if $x \in Q$ let $x \beta=x \gamma$. If $x \in R$ then either (i) $x\left(\alpha^{\circ} \alpha^{-1}\right) \cap P \neq \varnothing$ in which case we choose $y$ in $x\left(\alpha^{\circ} \alpha^{-1}\right) \cap P$, or (ii) $x\left(\alpha^{\circ} \alpha^{-1}\right) \cap$ $P=\varnothing$ and $x\left(\alpha^{\circ} \alpha^{-1}\right) \cap Q \neq \varnothing$, in which case we choose $y$ in $x\left(\alpha \circ \alpha^{-1}\right) \cap Q$. In either case $y \beta$ is already defined and we define $x \beta=y \beta$.

We show that $\beta^{2}=\alpha$. Let $x \in P$. Then the definition of $\Delta_{\mathrm{A}}$ makes it clear that 
$x \Delta_{A} \in P$, and so $x \beta^{2}=x \Delta_{A}^{2}$. But the construction of $\Delta_{A}$ entails that $x \Delta_{A}^{2}=x \alpha$ for all $x$ in $P=\operatorname{dom} \Delta_{\mathrm{A}}$.

Now let $x \in Q$, so that $x \beta=x \gamma$. Then $x \gamma \in \operatorname{stran} \alpha$. We shall show that $x \gamma \in Q$. For suppose by way of contradiction, that $x \gamma \in P$. Then there exists $z$ in Bas $\alpha \cup(\operatorname{Bas} \alpha) \Delta$ such that $z \alpha^{r}=x \gamma$. Since $z \alpha^{r} \in \operatorname{stran} \alpha$ we must have $r \geqslant i(z)$ and so

$$
\begin{aligned}
x \alpha=\left(z \alpha^{r}\right) \gamma & =\left(z \alpha^{i(z)+r-i(z)}\right) \gamma \\
& =z \alpha^{i(z)} \gamma \alpha^{r-i(z)}
\end{aligned}
$$

Now the element $z$ has a dual $z \Delta$, and $\left(z \alpha^{i(z)}\right) \gamma$ is equal either to $(z \Delta) \alpha^{i(z \Delta)}$ (rule (D1) or (D3)) or to $(z \Delta) \alpha^{i(z \Delta)+1}$ (rule (D2) or (D4)). Hence either

$$
x \alpha=(z \Delta)^{i(z \Delta)+r-i z} \quad \text { or } \quad x \alpha=(z \Delta)^{i(z \Delta)+r-i(z)+1},
$$

and in either case $x \alpha \in P$. Hence $x \alpha^{\prime} \in P$ for $t=1,2, \ldots$ But since $x \in \operatorname{stran} \alpha$ we have that $x \alpha^{t}=x$ for some $t \geqslant 1$. Hence $x \in P$, contrary to assumption. We conclude that $x \gamma \in Q$, and it is now obvious that

$$
x \beta^{2}=x \gamma^{2}=x \alpha .
$$

Finally, suppose that $x \in R$. Then $x \beta=y \beta$ where $y \in P \cup Q$ and $y \alpha=x \alpha$. Hence

$$
x \beta^{2}=y \beta^{2}=y \alpha=x \alpha
$$

in this case also. This completes the proof of Theorem 7.

REMARK. The criterion we have established for the existence of a square root is disappointingly complicated, but for small sets $X$ is not too hard to apply. Moreover, as the proof makes clear, the permutation $\gamma$ and the partial mapping $\Delta_{\mathrm{A}}$ do actually in effect construct the square root.

If one visualises elements of $\mathscr{T}(X)$ as directed graphs it is possible to view the problem as one of finding square roots (in a natural sense) for a certain kind of digraph. The only reference we have found on this topic [1] (for which we are indebted to Dr Ian Anderson) does not seem to be very helpful in our algebraic context.

\section{REFERENCES}

1. Dennis P. Geller, The square root of a digraph, J. Combin. Theory 5 (1968) 320-321.

2. J. M. Howie, An introduction to semigroup theory (Academic Press, 1976).

3. R. Isaacs, Iterates of fractional order, Canad. J. Math. 2 (1950) 409-416.

4. Mary Snowden, Square roots in full transformation semigroups, M.Sc. thesis, University of St Andrews (1979).

Four PEAKS

Clayton Road

Mold, Clwyd

WALES

Mathematical Institute

UNIVERSITY OF ST ANDREWS 